\title{
Measurement of the degree of salinity of water with a fiber-optic sensor
}

\author{
Óscar Esteban, Maria Cruz-Navarrete, Agustín González-Cano, and Eusebio Bernabeu
}

\begin{abstract}
A fiber-optic sensor based on surface-plasmon resonance for the determination of the refractive index is used for measuring the degree of salinity of water. The transducing element consists of a multilayer structure deposited on a side-polished monomode optical fiber. Measuring the attenuation of the power transmitted by the fiber shows that a linear relation with the refractive index of the outer medium of the structure is obtained. The system is characterized by use of a varying refractive index obtained with a mixture of water and ethylene glycol. Experimental results show that the sensor can be used as a salinity-degree measurement device with environmental applications. (C) 1999 Optical Society of America

OCIS codes: $\quad 010.4450,010.7340,060.2370,120.4630,240.6680,310.2790$.
\end{abstract}

\section{Introduction}

In recent years, fiber-optic sensors have experienced rapid development, and their field of application has been increased considerably. ${ }^{1}$ These devices are extremely versatile and provide nondestructive testing while maintaining a high level of accuracy and a relatively low cost. For these reasons, their use in natural environments to control different parameters of biological interest is nowadays quite extensive. In many cases such wide use results from designing integrated systems of several fiber-optic sensors that can measure with different accuracies or dynamic ranges a series of physical or chemical parameters.

A well-known type of fiber-optic sensor that has been applied for above goal is based on surfaceplasmon resonance in thin metallic films deposited on side-polished fibers. ${ }^{2}$ One of the parameters that has been measured by use of this technique is the refractive index of liquid media. ${ }^{3}$

In this paper we propose an application of these refractive-index sensors to determine the degree of salinity of water. This is a parameter of great biological and environmental interest, and its relation

The authors are with the Departamento de Óptica, Facultad de Ciencias Físicas, Universidad Complutense, Ciudad Universitaria, 28040 Madrid, Spain. A. González-Cano's e-mail address is agus@fis.ucm.es.

Received 17 February 1999; revised manuscript received 21 May 1999.

0003-6935/99/255266-05\$15.00/0

(C) 1999 Optical Society of America with the refractive index has been established empirically. Traditionally, the salinity degree is determined by the measurement of conductivity, but an optical measurement presents some advantages, not only because of the good properties of fiber-optic sensors but also because of the possibility of integration in the above-mentioned systems of sensors.

The physical principle of the sensor is the attenuation of the power guided by a fiber that results from the excitation of surface plasmons in a multilayer structure deposited on the fiber. Experimental results show that, by selection of the parameters of the layers, the response of the transducer can be adjusted so that linear behavior in the region of interest (i.e., a refractive index close to 1.33 ) is obtained. The salinity degree is then determined with an empirical algorithm. The sensor is small, inexpensive, and stable and can be used potentially as an in situ realtime controller of the degree of salinity.

\section{Theoretical Foundations}

Surface plasmons in thin metallic layers can be excited by light that is guided by an optical fiber if the fiber is side polished and the metallic layer is deposited onto it, thus producing a coupling with the evanescent field of the guided mode. This effect reduces the power transmitted by the fiber, and this attenuation is strongly dependent on the refractive index of the external medium that is in contact with the metallic layer. In this way any change in that index, for instance, one produced by the presence of some chemical component in that medium, induces a 


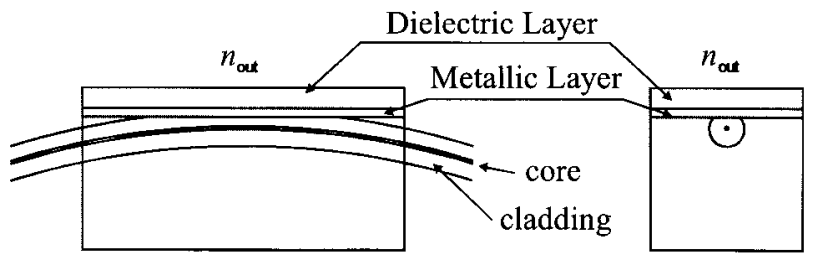

Fig. 1. Schematic of the sensing device. A fiber is polished, and a multilayer structure is deposited near the core.

change in the power transmitted through the fiber and can be measured.

Theoretical models ${ }^{4,5}$ have been proposed that are based on the interaction of the modes that can be guided by the structure of the plane layers and the modes guided by the fiber. When a multilayer structure is deposited on the fiber an effective refractive index for the whole structure that depends on the thickness of the layers and their refractive indices (including that of the external medium) is calculated. Maximum coupling (and minimum transmitted power) is predicted for a refractive-index value of the external medium that makes the effective index of the structure coincident with the effective propagation index of the mode guided by the fiber. The sharpness of that minimum and its location depend on the parameters of the structure. Some authors have reported that these models do not respond adequately to the phenomenology and a displacement of the position of the minimum seems to appear systematically. Some modifications of the models have been proposed for better adjustment to the experimental results. ${ }^{3}$

It is important to add that the only polarization mode of the fiber that can excite surface plasmons is the transverse-magnetic mode (the electric vector is perpendicular to the layers), so a strong dependence of the attenuation on the polarization occurs. This means that, in any practical application, polarization must be controlled to achieve stable behavior.

\section{Experimental Setup}

For preparation of the transducers the procedure depicted by Alonso et al. ${ }^{3}$ was used. Layers are deposited by thermal evaporation under high-vacuum conditions on a curved monomode fiber that has been polished (Fig. 1) until the cladding has a thickness of some micrometers. A thin metallic layer is then deposited, and a slightly thicker dielectric film is superposed on it. Thus the external medium is not in direct contact with the metallic layer. The presence of the dielectric is important to permit an adjustment of the dynamic range of the sensor by displacement of the location of the minimum of the transmitted power.

We used aluminum for the metallic layer, and we tested two different layer thicknesses: 8 and $12 \mathrm{~nm}$. This layer thickness also determines the dynamic range, and we proved that the 8-nm layer thickness is best suited for our application (with refractive indices

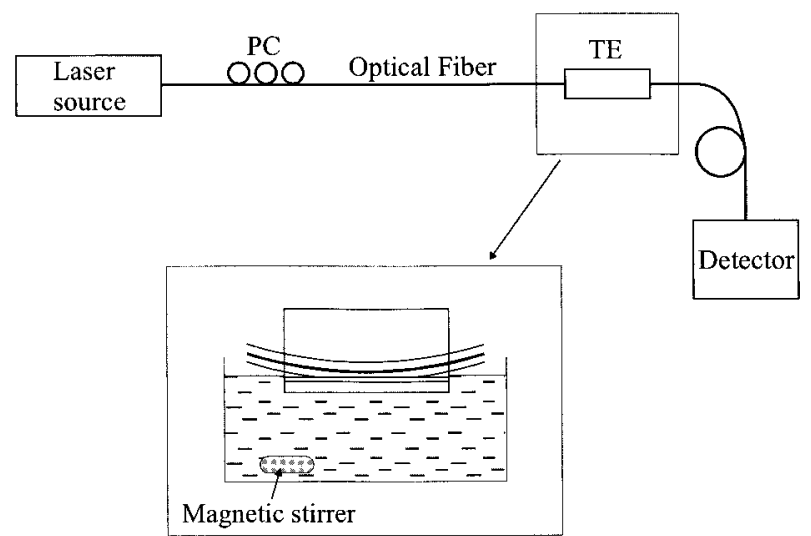

Fig. 2. Experimental setup. PC, polarization controller; TE, transducing element.

of the external medium close to 1.33). The dielectric material was $\mathrm{TiO}_{2}$, and the tested thicknesses were 54 and $56 \mathrm{~nm}$. The best results were obtained with the latter thickness.

The experimental setup used for both characterization of the sensor and salinity measurements is shown in Fig. 2. Light is provided by a commercial laser diode with a wavelength of $780 \mathrm{~nm}$. The laser is pigtailed into a silica monomode optical fiber that is connected to the fiber that contains the transducer. This fiber (3M Company, Model FS-SN 4224) is monomode at $820 \mathrm{~nm}$, and its core has a diameter of $4.7 \mu \mathrm{m}$. A polarization controller is introduced between the source and the transducer to ensure that the polarization of the light incident upon the transducer is in the transverse-magnetic mode. The output power of the laser is monitored. Light exiting the fiber is detected by a silicon photodiode (HewlettPackard, Model HP 8152A Optical Average Power Meter). Also, the temperature is determined during the measurement because a significant dependence of the refractive index on the temperature exists.

\section{Characterization}

To characterize the sensor, we used a mixture of distilled water and chemically pure ethylene glycol. The refractive index of the mixture depends on the concentration of ethylene glycol, according to the following empirical law6:

$$
n(T)=n_{\mathrm{H}_{2} \mathrm{O}}(T)+0.111 \frac{V_{\text {eth }}}{V_{\text {tot }}},
$$

where $n$ is the refractive index of the mixture, $n_{\mathrm{H}_{2} \mathrm{O}}$ is the refractive index of water, $V_{\text {eth }}$ is the volume of ethylene glycol, and $V_{\text {tot }}$ is the total volume of the mixture. Both refractive indices depend on the temperature, and this fact is taken into account in the calculation. Ethylene glycol presents some advantages for characterization, mainly because of the high value of its refractive index, and provides a wide range of refractive indices when its concentration is varied. However, some difficulties arise when it has 


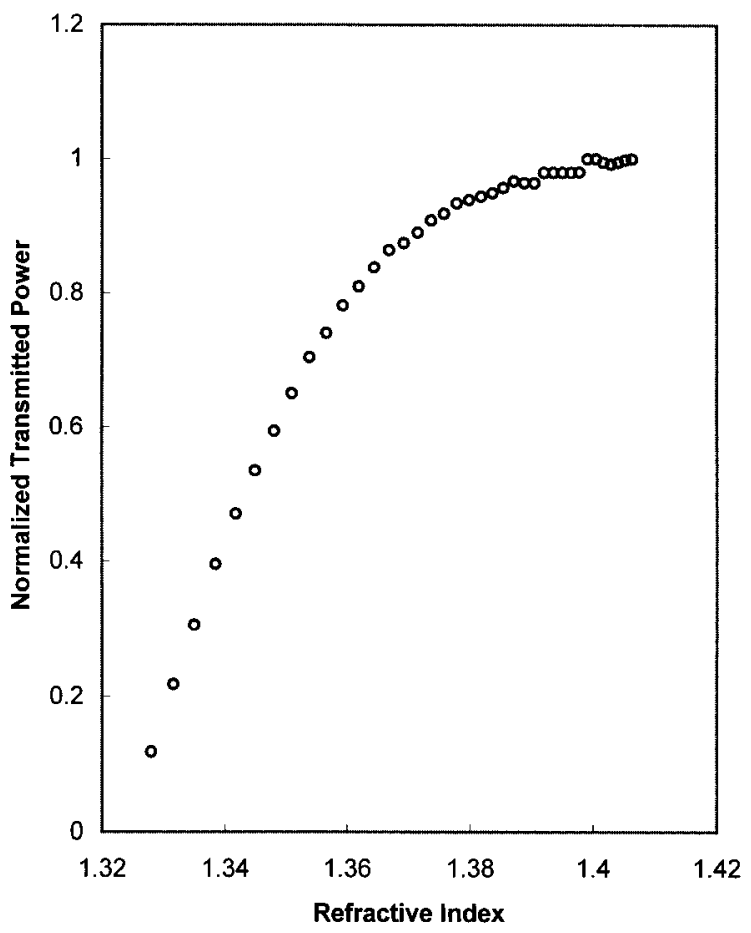

Fig. 3. Characterization of the sensor. The normalized-output transmitted power is plotted versus the refractive index of a mixture of water and ethylene glycol.

to be mixed with water, and efforts must be made to obtain a homogeneous mixture. A magnetic stirrer is used for that purpose.

We tested a range of refractive indices from 1.327 (pure water) to 1.41. This range is wider than the region of interest for salinity measurements, but we can, in this way, obtain a realistic picture of the behavior of the sensor outside of this region. This behavior is qualitatively, although not quantitatively, predicted by the theoretical models outlined in Section 2.

The results of the characterization are shown in Figs. 3 and 4. In Fig. 3 the complete range of refractive indices is shown. Linear behavior is exhibited in the region of interest and can be seen in detail in Fig. 4. On the ordinate axis, we show the normalized-output transmitted power. Values of the transmitted power were divided by the maximum obtained value and correspond to the power transmitted by the system when the external medium has a high refractive index. These results show that this particular sensor is well suited for salinity measurements because the refractive indices that can arise in these measurements are close to 1.33 , as we said in Section 3.

\section{Determination of the Degree of Salinity}

The salinity degree $S$ of water is a parameter that measures the salt content of water in terms of the concentration of chlorine ions. It is usually measured by the determination of the electrical conductivity. The range of degrees of salinity that we

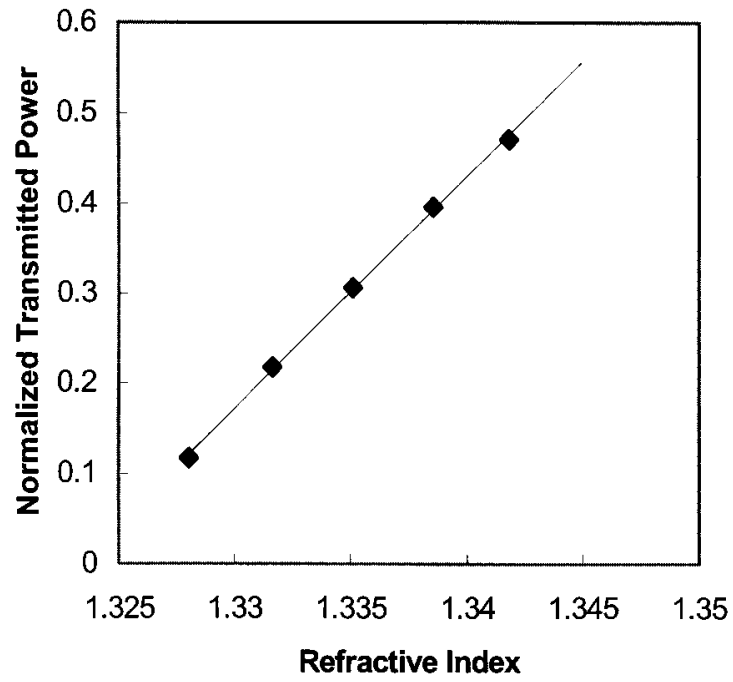

Fig. 4. Detail of the curve of Fig. 3 showing the linear behavior of the sensor within the range of refractive indices of interest for salinity measurements.

measured extends from zero (pure water) to $40 \%$ of the concentration of chlorine ions. A standard value for the salinity degree of sea water is $35 \% 0 .{ }^{7}$

The empirical relation between the degree of salinity of water and the refractive index is well established. Several algorithms have been proposed in the literature. Among them, we used that of Quan and Fry ${ }^{8}$ (see Appendix A). With this algorithm, we can, in principle, convert the degree of salinity into the refractive index and vice versa. We must note, however, that temperature is one of the parameters present in the algorithm, so we must control it and introduce it into the calculation. The variation from zero to $40 \%$ of the degree of salinity is equivalent to variation from 1.327 to 1.335 of the refractive index.

Experimentally, the salinity degree of water was varied gradually by the addition of commercial artificial salt (Aquarium System, Instant Ocean) to pure water in a controlled manner. The degree of salinity of the mixture was measured continuously with a conductivity meter (Orion, Model 162), so we can measure the response of the system for a series of values of the salinity degree with an accuracy of $0.1 \%$. We obtained a linear relation between the degree of salinity of water and the response of the system, as is shown in Fig. 5. The correlation coefficient is high enough (typically, 0.999) to prove the suitability of this procedure as a method for the determination of the degree of salinity. For the graph of Fig. 5, we made a temperature correction to permit us to represent the pure dependence on salinity. Details are given in Appendix A.

On the other hand, we can calculate, with the above-mentioned algorithm, the values of the refractive indices that correspond to the different salinity degrees and obtain a curve that relates the refractive index of salt water to the transmitted power. The results obtained are in good agreement with the characterization depicted in Section 4. This agreement 


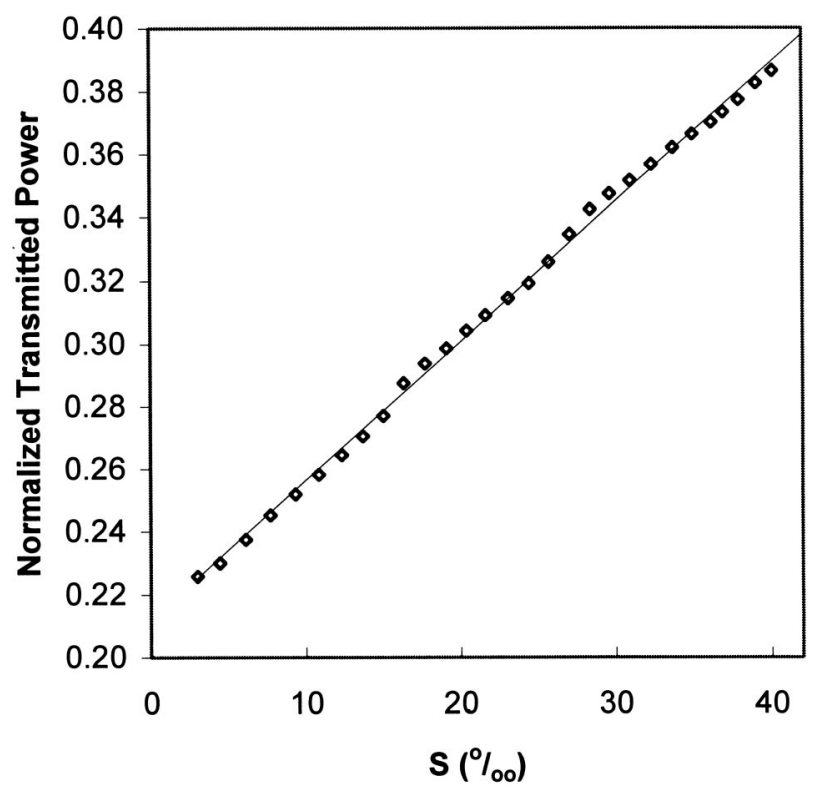

Fig. 5. Experimental results obtained for the degree of salinity. The temperature correction, as explained in the text, was performed. The reference temperature is $25^{\circ} \mathrm{C}$. A linear regression curve was added.

shows that calibration of the sensor to the refractive index is sufficient to permit salinity-degree measurements. In principle, the curves in Fig. 5 show that the accuracy of the system is high enough to provide a salinity-degree determination to within $\pm 0.1 \%$, which is the accuracy of the conductivity meter.

\section{Conclusions}

We have applied a refractive-index optical sensor based on surface-plasmon excitation to the measurement of the degree of salinity of water, proving in this way the suitability of the device for that type of measurement. The response is linear, the results are repeatable, and the accuracy is adequately high. The calibration of the device is simple, and any mixture of liquids with variable refractive indices can be used. The degree of salinity is obtained in an absolute way, provided we know the response for any reference, such as pure water. Although this paper shows only preliminary results that prove the suitability of our sensor, a comparison can be made with commercial conductivity meters that shows that a fiber-optics approach can be competitive with those systems.

A fiber-optic sensor presents, as has been stated, several advantages: It is as simple and as small as a conductivity meter, it is inexpensive, and it provides nondestructive measurements. Its main attractions are that the use of fiber-optics technology permits the integration of a general-purpose measuring system for monitoring seawater and that access to difficult places can be made easier. Although at this stage of development the reliability of the sensor is not yet the same as that of a conductivity meter (mainly because of problems of repeatability associ- ated with the stability of the source), further developments can improve this aspect. The results presented in this paper prove that this type of device can, in principle, be used as the basis of an in situ real-time controller of the degree of salinity in places of interest ecologically.

\section{Appendix A: Algorithm for the Determination of the Degree of Salinity}

To transform a refractive-index measuring sensor to a salinity-degree sensor, we need a relation between the magnitudes of both refractive index and salinity. This was provided in a paper by Quan and $\mathrm{Fry}^{8}$ and was based on extensive empirical results. The algorithm is given by the following formula:

$$
\begin{aligned}
n(S, T, \lambda)= & 1.31405+\left(1.779 \times 10^{-4}-1.05 \times 10^{-6} T\right. \\
& \left.+1.6 \times 10^{-8} T^{2}\right) S-2.02 \times 10^{-6} T^{2} \\
& +\frac{15.868+0.01155 S-0.00423 T}{\lambda} \\
& -\frac{4382}{\lambda^{2}}+\frac{1.1455 \times 10^{6}}{\lambda^{3}},
\end{aligned}
$$

where $S$ is the salinity degree in thousandths $T$ is the temperature in degrees Celsius, and $\lambda$ is the wavelength in nanometers.

It can be seen from Eq. (A1) that the refractive index appears to show dependence on several parameters that can vary during experiments. In principle, we do not consider a variation of wavelength because we are using a laser, but both the degree of salinity and the temperature can vary. Because we controlled both parameters during the measurements, we performed a so-called temperature correction to represent the behavior of the sensor. To control the response with the salinity degree, we transformed the value of the degree of salinity to the value that it should have for a constant temperature of $25^{\circ} \mathrm{C}$ to yield the same refractive index, according to Eq. (A1). This correction is made only to permit the representation (where we show only the dependence of the response of the sensor on the variation of the degree of salinity) of the sensor because it was difficult to achieve a strictly constant temperature during the measurements. However, realizing constant temperature should not be a problem with real measurements as long as we can control the temperature (or, at least, assume that it is essentially constant) and simultaneously take the value of the temperature as an input to the algorithm.

We wish to thank to R. Alonso, C. Cosculluela, J. Pelayo, and F. Villuendas of the Department of Applied Physics, University of Zaragoza, Spain, for their support and for providing us with the sensors used for this work. This research has been supported by the European Union under Project SOFIE (spectroscopy using optical fibers in the marine environment), number MAS3-CT97-0157. 


\section{References}

1. J. Dankin, Optical Fiber Sensors (Artech House, Norwood, Mass., 1989).

2. S.-M. Tseng and C.-L. Chen, "Side-polished fibers," Appl. Opt. 31, 3438-3447 (1992).

3. R. Alonso, F. Villuendas, J. Tornos, and J. Pelayo, "New 'in-line' optical-fibre sensor based on surface plasmon excitation," Sens. Actuators A 37-38, 187-192 (1993).

4. D. Marcuse, "Investigation of coupling between a fiber and an infinite slab," J. Lightwave Technol. 7, 122-130 (1989).

5. A. Tz. Andreev and K. Panajotov, "Distributed single-mode fiber to single-mode planar waveguide coupler," J. Lightwave Technol. 11, 1985-1989 (1993).

6. R. Alonso, "Estudio teórico y experimental de dispositivos ópticos basados en el acoplamiento entre el modo guiado por una fibra óptica y estructuras multicapa incluyendo medios metálicos," Ph.D. dissertation (Universidad de Zaragoza, Spain, 1995).

7. K. S. Shifrin, Physical Optics of Ocean Water (American Institute of Physics, New York, 1988).

8. X. Quan and E. S. Fry, "Empirical equation for the index of refraction of seawater," Appl. Opt. 34, 3477-3480 (1995). 